\title{
O uso da CIF no contexto escolar inclusivo: um mapeamento bibliográfico
}

\author{
Using ICF in the special education: a bibliographic mapping
}

El uso del CIF en el contexto escolar inclusivo: un mapeo bibliográfico

Mariângela Castilho Uchoa de Oliveira

Mestra pela Universidade Presbiteriana Mackenzie, São Paulo, SP, Brasil

E-mail: maricastilho09@gmail.com ORCID: https://orcid.org/0000-0002-5844-0610

Camila Miccas

Professora doutora do Centro Universitário Sumaré e da rede estadual de São Paulo, São Paulo, SP, Brasil E-mail: camila_miccas@hotmail.com ORCID: https://orcid.org/0000-0002-9961-7996

Catherine Oliveira de Araújo

Professora doutora do Centro Universitário Adventista de São Paulo, São Paulo, SP, Brasil

E-mail: cathearaujo22@hotmail.com ORCID: https://orcid.org/0000-0003-2031-9302

\section{Maria Eloisa Famá D'Antino}

Professora doutora da Universidade Presbiteriana Mackenzie, São Paulo, SP, Brasil

E-mail: eloidantino@gmail.com ORCID: https://orcid.org/0000-0002-7072-4755

Recebido em 04 de março de 2020

Aprovado em 08 de março de 2021

Publicado em 23 de março de 2021

\section{RESUMO}

A Classificação Internacional de Funcionalidade, Incapacidade e Saúde (CIF) é uma classificação desenvolvida pela Organização Mundial de Saúde (OMS). A partir de uma revisão da Classificação Internacional de Deficiências, Incapacidades e Desvantagens (CIDID), fez-se uma mudança de contexto, do enfoque negativo da deficiência e incapacidade para uma perspectiva positiva com ênfase nas atividades e na participação do indivíduo com alterações de função e/ou da estrutura do corpo (FARIAS; BUCHALLA, 2005). Esta classificação apresenta diferentes objetivos, sendo que, primordialmente pode oferecer um olhar sobre a funcionalidade e incapacidade global do indivíduo, assim como padronizar informações a serem utilizadas por diferentes profissionais, tais como: fisioterapeutas, fonoaudiólogos, professores, entre outros. Nesta padronização de informações, pode-se incluir o ambiente escolar. Este estudo teve como objetivo mapear publicações sobre o uso da CIF em ambiente escolar inclusivo. O método adotado foi uma revisão da literatura de caráter retrospectivo, em três indexadores de produção científica: Scielo, Lilacs e Pubmed. Resultou dessa busca 91 artigos, e, após a leitura dos resumos, 16 artigos preenchiam os critérios e foram lidos na íntegra. Verificou-se que há escassez de publicações sobre o tema e constatou-se a necessidade de novos estudos que contemplem esta temática. Uma vez que a CIF não classifica as pessoas, mas descreve a sua funcionalidade e incapacidade, é necessário que os profissionais inseridos no contexto escolar a dominem a fim de promover programas e políticas que respondam eficientemente 
http://dx.doi.org/10.5902/1984686X42725

às demandas de aprendizagem e desenvolvimento do indivíduo com deficiência, ampliando sua participação em atividades escolares e sociais.

Palavras-chave: CIF; Classificação Internacional de Funcionalidade; Inclusão Escolar.

\section{ABSTRACT}

The International Classification of Functioning, Disability and Health Is a classification developed by the World Health Organization (WHO), that after a review of the International Classification of Disabilities and Disadvantages (ICIDH), a change of context was made from the negative focus on disability to a positive perspective with emphasis on the activities and participation of the individual with disabilities in his function/ or body structure (FARIAS; BUCHALLA, 2005). There are different objectives, primarily being useful for functioning and global disability of the individual, just as the standardization of information that can be used by different professionals, such as physiotherapist, speech therapist, teachers, and others. In this standardization of information, we can include the school environment. This study aimed to map the publications on the use of ICF in an inclusive school environment. The method adopted was a review of the retrospective literature in three scientific science indexers: Scielo, Lilacs and Pubmed. It resulted in 91 articles, however after reading the abstracts, only 16 articles filled the criteria and were read in full. It was verified that there is a shortage of publications on this subject and it was noted the need of new studies that contemplate this subject. Since ICF does not classify people, but their functioning and incapacity, it is necessary that professionals inserted in the school context dominate it in order to promote programs and policies that respond efficiently to the learning and development demands of the individual with disabilities, expanding their participation in school and social activities.

Keywords: ICF; International Classification of Functioning; School Inclusion.

\section{RESUMEN}

La Clasificación Internacional de Funcionamiento, Discapacidad y Salud (CIF) es una clasificación desarrollada por la Organización Mundial de la Salud (OMS). A partir de una revisión de la Clasificación Internacional de Deficiencia, Discapacidades y Minusvalía (CIDDM), se ha realizado un cambio de contexto, del enfoque negativo sobre la discapacidad ha sido reemplazado a una perspectiva positiva con énfasis en las actividades y la participación del individuo con cambios en la función y/o estructura corporal (FARIAS; BUCHALLA, 2005). Esta clasificación tiene diferentes objetivos y puede analizar principalmente la funcionalidad y la discapacidad general del individuo, así como también estandarizar la información para ser utilizada por diferentes profesionales, como fisioterapeutas, logopedas, maestros, entre otros. Esta estandarización de la información puede incluir el entorno escolar. Este estudio tuvo como objetivo mapear publicaciones sobre el uso del CIF en un entorno escolar inclusivo. El método adoptado fue una revisión bibliográfica retrospectiva en tres indexadores de producción científica: Scielo, Lilacs y Pubmed. Esta búsqueda resultó en 91 artículos, y después de leer los resúmenes, 16 artículos cumplieron con los criterios y se leyeron en su totalidad. Se descubrió que hay una escasez de publicaciones sobre el tema y se observó la necesidad de realizar más estudios 
que lo aborden. Dado que la CIF no clasifica a las personas, sino que describe su funcionalidad y discapacidad, es necesario que los profesionales insertados en el contexto escolar lo dominen para promover programas y políticas que respondan de manera eficiente a las demandas de aprendizaje y desarrollo del individuo con discapacidad, ampliando su participación en las actividades escolares y sociales.

Palabras clave: CIF; Clasificación Internacional de Funcionalidad; Inclusión Escolar.

\section{Introdução}

A Organização Mundial da Saúde (OMS) tem hoje duas classificações de referência para descrição do estado de saúde: a Classificação Estatística Internacional de Doenças e Problemas Relacionados à Saúde, que corresponde à décima revisão da Classificação Internacional de Doenças (CID-10, 1990) e a Classificação Internacional de Funcionalidade, Incapacidade e Saúde (CIF, OMS, 2001). A CIF investiga várias dimensões da vida dos indivíduos avaliados, levando em consideração, ao documentar a funcionalidade, não apenas aspectos da saúde, mas também culturais, políticos e sociais. Deste modo, à medida que a utilização da CIF passa a ser realizada por um número maior de profissionais em diferentes contextos, os resultados acerca da funcionalidade serão mais abrangentes $\mathrm{e}$ mais fidedignos (DI NUBILA; BUCHALLA, 2008).

A CIF tem diferentes objetivos, sendo que, primordialmente, pode oferecer um olhar para o desenvolvimento e a funcionalidade global do indivíduo, assim como padronizar informações a serem utilizadas por diferentes profissionais. É uma classificação ampla que pretende ser clara em relação ao seu objeto de estudo. Em seu escopo pode-se englobar todos os aspectos da saúde humana e componentes relacionados ao bem-estar, estes últimos denominados domínios, e incluem, por exemplo, educação, mobilidade e interações sociais. Já os domínios de saúde incluem aspectos como ver, ouvir, andar, aprender e lembrar (OMS, 2008).

As propriedades da CIF são organizadas em duas partes: (1) a de Funcionalidade e Incapacidade, (2) Fatores Contextuais. Cada parte tem dois componentes:

1. Componentes da Funcionalidade e da Incapacidade - que inclui o componente Corpo com duas classificações, uma para as funções dos sistemas orgânicos e outra para as estruturas do corpo. E o componente Atividades e Participação que contempla a faixa completa de domínios que indicam os aspectos da funcionalidade, tanto na perspectiva individual como social. 
http://dx.doi.org/10.5902/1984686X42725

2. Componentes dos Fatores Contextuais - O primeiro componente dos Fatores Contextuais é uma lista de Fatores Ambientais. Estes têm um impacto sobre todos os componentes da funcionalidade e da incapacidade e estão organizados de forma sequencial, do ambiente mais imediato do indivíduo até o ambiente geral. Os Fatores Pessoais também são um componente dos Fatores Contextuais, mas eles não estão classificados na CIF devido à grande variação social e cultural associada aos mesmos.

Figura 1 - Modelo integrador de funcionalidade humana

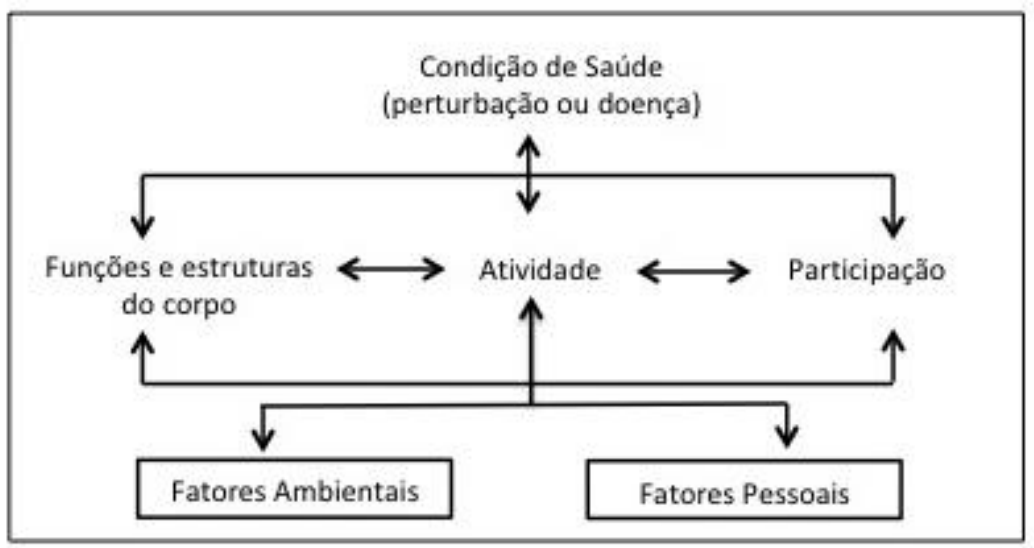

Fonte: CIF, EDUSP, 2003, p. 32.

Figura 2 - Uma visão geral da CIF

\begin{tabular}{|c|c|c|c|c|}
\hline \multirow[b]{2}{*}{ Componentes } & \multicolumn{2}{|c|}{ Parte 1: Funcionalidade e Incapacidade } & \multicolumn{2}{|c|}{ Parte 2: Fatores Contextuais } \\
\hline & $\begin{array}{c}\text { Funções e Estruturas } \\
\text { do Corpo }\end{array}$ & $\begin{array}{l}\text { Atividades e } \\
\text { Participação }\end{array}$ & $\begin{array}{c}\text { Fatores } \\
\text { Ambientais }\end{array}$ & Fatores Pessoais \\
\hline Dominios & $\begin{array}{l}\text { Funções do Corpo } \\
\text { Estruturas do Corpo }\end{array}$ & $\begin{array}{c}\text { Áreas Vitais } \\
\text { (tarefas, açőes) }\end{array}$ & $\begin{array}{c}\text { Influências externas } \\
\text { sobre a } \\
\text { funcionalidade e a } \\
\text { incapacidade }\end{array}$ & $\begin{array}{l}\text { Influências internas } \\
\text { sobre a } \\
\text { funcionalidade e a } \\
\text { incapacidade }\end{array}$ \\
\hline Constructos & $\begin{array}{l}\text { Mudança nas funções do } \\
\text { corpo (fisiológicas) } \\
\text { Mudança nas estruturas } \\
\text { do corpo (anatômicas) }\end{array}$ & $\begin{array}{c}\text { Capacidade } \\
\text { Execução de tarefas num } \\
\text { ambiente padrão } \\
\text { Desempenho/ Execução } \\
\text { de tarefas no ambiente } \\
\text { habitual }\end{array}$ & $\begin{array}{l}\text { Impacto facilitador } \\
\text { ou limitador das } \\
\text { caracteristicas do } \\
\text { mundo fisico, social e } \\
\text { atitudinal }\end{array}$ & $\begin{array}{c}\text { Impacto dos } \\
\text { atributos de uma } \\
\text { pessoa }\end{array}$ \\
\hline \multirow{2}{*}{ Aspectos Positivos } & $\begin{array}{c}\text { Integridade funcional e } \\
\text { estrutural }\end{array}$ & $\begin{array}{l}\text { Atividades } \\
\text { Participação }\end{array}$ & \multirow{2}{*}{ Facilitadores } & \multirow{2}{*}{ Nâo aplicável } \\
\hline & \multicolumn{2}{|c|}{ Funcionalidade } & & \\
\hline \multirow[t]{2}{*}{ Aspectos Negativos } & Deficiência & $\begin{array}{l}\text { Limitação da atividade } \\
\text { Restrição da participação }\end{array}$ & \multirow[t]{2}{*}{ Barreiras } & \multirow[t]{2}{*}{ Não aplicável } \\
\hline & \multicolumn{2}{|c|}{ Incapacidade } & & \\
\hline
\end{tabular}

Fonte: CIF, EDUSP, 2003, p. 24. 
http://dx.doi.org/10.5902/1984686X42725

As funções do corpo são as funções fisiológicas do sistema corpóreo, já as estruturas do corpo são as partes anatômicas do corpo, como órgãos, membros e seus componentes. As deficiências são as alterações nas funções ou nas estruturas. A atividade é entendida como a execução de tarefas, isto é, a perspectiva individual de funcionalidade. A participação é definida como o envolvimento do indivíduo em uma situação da vida, corresponde à perspectiva social da funcionalidade. Diferente uma da outra, limitação de atividade é quando o indivíduo encontra dificuldades para realizar uma atividade, como andar, e restrições às participações são os problemas que este indivíduo pode enfrentar em sua vida diária (RIBERTO, 2011).

ACIF é referência para a Legislação internacional e nacional sobre os direitos humanos, sendo utilizada por setores, como: economia, educação, previdência social, seguros, trabalho, política social e legislação ambiental.

Conforme Ruaro et al. (2012), a CIF tem sido apontada como uma ferramenta multidimensional que visualiza a saúde humana sob diversas vertentes e abordagens, podendo ser usada nos mais variados setores, incluindo a educação. Sua utilização deve ser implementada em práticas clínicas, no ensino e em pesquisas, embora trabalhos que embasam o seu uso ainda sejam escassos no Brasil.

A CIF apresenta foco na funcionalidade dos sujeitos com o intuito de trazer à luz as capacidades e potencialidades dos sujeitos avaliados, e surgiu pela necessidade de se avaliar, numa perspectiva biopsicossocial, diversos aspectos da vida de indivíduos, com o objetivo de uniformizar informações para que diferentes profissionais padronizem relatórios que sejam utilizados em diferentes áreas, tais como a educação, a saúde e os serviços sociais (CASTRO; PINTO, 2012).

Por seu enfoque biopsicossocial, a CIF prioriza a funcionalidade do sujeito como componente da saúde, visando conhecer, além de suas limitações, dificuldades e comprometimentos em atividades e participação, também as condições que lhes são oferecidas, como facilitadoras ou dificultadoras do funcionamento de cada sujeito. Possui uma estrutura conceitual multidimensional, o que permite ser uma forma de coletar e organizar informações do indivíduo quanto ao seu funcionamento e envolvimento nas situações de vida, levando em consideração o contexto (BRASILEIRO et al., 2009; ADOLFSSON; FLEISCHER, 2015).

Pela perspectiva da CIF, a funcionalidade humana tem grande potencial de se tornar uma área de pesquisa multifacetada, onde pesquisadores de formações variadas podem 
http://dx.doi.org/10.5902/1984686X42725

associar seus conhecimentos com o intuito de melhorar a participação das pessoas com deficiência (STUCKI et al., 2008). Os estados de saúde, que são as doenças, transtornos, dentre outros, são classificados pela CID-10, enquanto a CIF classifica as funcionalidades e incapacidades destes mesmos estados de saúde. Uma classificação não pode substituir a outra, elas se complementam e devem ser utilizadas em conjunto (DI NUBILA, 2010).

Sendo assim, a CIF não classifica pessoas e nem conclui diagnóstico de doença, mas, é capaz de descrever a situação que cada indivíduo apresenta em diversos domínios, fundamentada numa abordagem ecológica e interdisciplinar, além de identificar as barreiras ambientais que são os obstáculos e/ou os facilitadores do processo. É um modelo inovador quando comparado com modelos onde aspectos biomédicos eram evidenciados ou ainda com os modelos sociais que focavam apenas nas estruturas e instituições sociais (PORTUGAL, 2008).

A CIF não substitui as ferramentas usualmente utilizadas para medir os aspectos da funcionalidade e do ambiente, mas identifica e qualifica a situação, diagnosticada por vários meios, usando uma linguagem comum que padroniza os conceitos e a nomenclatura (ARAÚJO; BUCHALLA, 2015).

Em uma perspectiva social, a incapacidade apresentada pelo indivíduo é compreendida como resultado de um conjunto de condições geralmente criadas pelo ambiente social, não podendo ser considerada, portanto, apenas um atributo do indivíduo, mas em relação às oportunidades ou limitações que o universo social proporciona. Sendo assim, a inclusão é uma ação de responsabilidade social coletiva para que aconteça uma reestrutura ambiental para a plena participação das pessoas com deficiência, em qualquer situação da vida social.

Desse modo, lembramo-nos dos estudos de Sekkel (2003) que concebe a educação inclusiva na perspectiva de um ambiente inclusivo, definindo-o como:

\footnotetext{
aquele que tem uma articulação coletiva e uma ação comprometida com o reconhecimento e busca da satisfação das necessidades de cada um, a qual se inscreve no âmbito da construção de uma sociedade verdadeiramente humana, em que as pessoas possam se diferenciar e se desenvolver em busca de felicidade (SEKKEL, 2003, p. 162).
}

A autora também sugere que a compreensão de um ambiente inclusivo amplia o foco da discussão da inclusão da criança com deficiência para todos os envolvidos: professores, profissionais de apoio, equipe técnica, pais, comunidade e a criança.

Acreditamos, assim, que a perspectiva de um ambiente inclusivo, proposto por Sekkel (2003), tem o seu respaldo na abordagem da CIF, que ressalta a funcionalidade do indivíduo, 
http://dx.doi.org/10.5902/1984686X42725

considerando sempre a relação de aspectos intrínsecos do sujeito com os do ambiente e das oportunidades que lhes são oferecidas.

Uma forma do aluno com deficiência ter as mesmas oportunidades em participar no contexto escolar com os demais alunos é promover a utilização da CIF no ambiente escolar. Esta classificação pode evidenciar as reais necessidades do aluno com deficiência, seja esta necessidade, uma mudança arquitetônica, conceitual ou ainda de recurso adicional, para que ele participe efetivamente das atividades em sua escola.

Atividade e Participação é o componente da CIF mais utilizado no contexto escolar. Segundo critérios da OMS (2003), atividade é descrita como execução de uma tarefa ou ação pelo indivíduo e participação é poder participar como os outros.

Este componente ajuda a descrever como o indivíduo é no seu dia a dia, como realiza suas atividades diárias e como participa da sua vida social, considerando todas as funções e estruturas do seu corpo. Todas as ações são consideradas, desde as ações mais simples até as mais complexas, incluindo domínios como: aprendizagem, comunicação, cuidados pessoais, demandas gerais, mobilidade, relações interpessoais, educação, trabalho e vida comunitária. As limitações de atividade são as dificuldades que o indivíduo pode apresentar para executar determinada tarefa, já os problemas que ele pode enfrentar ao se envolver em situações da vida, são consideradas restrições à participação (FARIAS; BUCHALLA, 2005).

Sabe-se que a avaliação no contexto escolar tem um caráter de identificação das condições de ensino e aprendizagem para orientação pedagógica e não diagnóstica. Assim, no transcorrer do processo educativo dos alunos com deficiência, a avaliação é realizada com o objetivo de identificar barreiras em diversas dimensões, considerando-se o aluno e o contexto educacional (VELTRONE; MENDES, 2011). Portanto, o uso dessas classificações pode fazer muito sentido no contexto escolar, até porque a legislação brasileira expressa que a avaliação pedagógica deve respeitar aspectos individuais do aluno, valorizando seus progressos em relação a si mesmo e em relação ao grupo em que está inserido (BRASIL, 2013).

A CIF pode ser grande aliada na avaliação de alunos com deficiência no ambiente escolar, uma vez que apresenta uma nova concepção de sujeito, privilegiando suas possibilidades de participação e interação com o ambiente, a despeito da condição intrínseca de deficiência. Assim, colocam o foco na condição de pessoa como um todo, e naquilo que cada indivíduo é capaz de desenvolver ou vir a desenvolver (MICCAS; D’ANTINO; 2011). 
http://dx.doi.org/10.5902/1984686X42725

Devido ao fato da CIF ter sido elaborada com o intuito de atender diferentes setores, apresenta alguns objetivos de maior especificidade que buscam responder às necessidades de diferentes áreas em que pode ser utilizada. Deste modo, de acordo com a OMS (2008), os objetivos específicos da CIF são: (1) proporcionar uma base científica para a compreensão e o estudo da saúde e seus determinantes, inclusive das condições relacionadas à saúde; (2) estabelecer uma linguagem comum para a descrição da saúde e dos estados relacionados à saúde para melhorar a comunicação entre diferentes usuários (profissionais de saúde, pesquisadores, elaboradores de políticas públicas e inclusive pessoas com deficiência); (3) permitir comparação de dados entre países, entre disciplinas relacionadas à saúde, entre serviços e em diferentes momentos ao longo do tempo; e fornecer um esquema de codificação para sistemas de informações de saúde.

Além de proporcionar uma avaliação biopsicossocial do aluno, o uso da CIF também permite coletar dados vitais sobre tipos e níveis de funcionalidade e incapacidade de modo consistente e comparável internacionalmente, fornecendo a base para compor dados em nível nacional, ajudando a guiar o desenvolvimento de políticas públicas em diferentes áreas (DI NUBILA, 2010), a promover a igualdade de oportunidades para todos e a apoiar a luta contra a discriminação das pessoas com deficiência ou incapacidade (DI NUBILA; BUCHALLA, 2008).

Uma classificação da funcionalidade torna-se extensa porque necessita abranger em seu sistema todas as condições de saúde, incapacidade e fatores que poderão interferir na funcionalidade do indivíduo avaliado, consequentemente, o processo de avaliação torna-se complexo e exaustivo, a menos que sejam criadas ferramentas de uso prático. De modo geral, clínicos utilizam $20 \%$ dos códigos da CIF para explicar uma determinada condição de saúde. Pensando nisso, a OMS, com o apoio de especialistas, criou listas e instrumentos baseados na CIF, denominados de Core Sets. Os Core Sets possuem categorias de itens em comum para uma determinada condição de saúde ou doença, podendo ainda ter itens adicionais, que dão uma visão mais detalhada sobre um determinado quadro clínico (RIBERTO, 2011).

A CIF se configura como importante instrumento de intercessão entre a área da saúde e da educação especial, podendo aproximar os profissionais das duas áreas no encaminhamento da avaliação funcional do indivíduo com deficiência e no planejamento de intervenções, orientados por uma perspectiva positiva da deficiência que enfatiza a 
http://dx.doi.org/10.5902/1984686X42725

atualização e ampliação das capacidades funcionais e a participação social do indivíduo, a partir do contexto da escola inclusiva.

\section{Método}

Esta pesquisa caracteriza-se como um mapeamento bibliográfico de artigos nacionais e internacionais referente à CIF no contexto escolar inclusivo, de caráter retrospectivo, realizado em três indexadores de produção científica: Lilacs (Literatura Latino-Americana e do Caribe em Ciências da Saúde, disponível em http://lilacs.bvsalud.org); PubMed (U.S. National Library of Medicine/National Institutes of Health, disponível em http:// www.ncbi.nlm.nih.gov/pubmed) e SciELO Brasil (Scientific Eletronic Library Online, disponível em http://www.scielo.br), utilizando os descritores abaixo mencionados:

Quadro 1 - Descritores

\begin{tabular}{|c|c|c|c|}
\hline Idioma & \multicolumn{3}{|c|}{ Descritores } \\
\hline Português & CIF Educação & CIF Educação Especial & CIF Inclusão Escolar \\
& CIF-CJ Educação & CIF-CJ Educação & CIF-CJ Inclusão Escolar \\
& Classificação & Especial & Classificação \\
& Internacional de & Classificação & Internacional de \\
& Funcionalidade & Internacional de & Funcionalidade Inclusão \\
& Educação & Funcionalidade & Escolar \\
\hline \multirow{3}{*}{ Espanhol } & Educação Especial & \\
& CIF-NJ Educación & CIF Educación Especial & CIF Inclusión Escolar \\
& Clasificación & CIF-NJ Educación & CIF-NJ Inclusión Escolar \\
& Internacional de & Especial & Clasificación \\
& Funcionalidad & Clasificación & Internacional de \\
& Educación & Internacional de & Funcionalidad Inclusión \\
& Funcionalidad Educación & Escolar \\
\hline Inglês & ICF Education & Especial & \\
& ICF-CY Education & ICF Special Education & ICF School Inclusion \\
& International & ICF-CY Special & ICF-CY School Inclusion \\
& Classification of & Education & International \\
& Functioning Education & International & Classification of \\
& & Classification of & Functioning School \\
& & Functioning Special & Inclusion \\
& & Education & \\
& & &
\end{tabular}

Fonte: elaborado pelas autoras (2018).

Como primeiro critério de inclusão, o trabalho deveria estar publicado no formato de artigo científico, sendo que as buscas incluíram artigos dos dez últimos anos.

Foram encontrados 91 artigos na busca inicial e selecionados 16 artigos que foram lidos na íntegra. Os 75 artigos excluídos não preencheram os critérios de inclusão. Os demais 
http://dx.doi.org/10.5902/1984686X42725

critérios de exclusão foram: teses, dissertações e monografias; artigos que apresentaram os descritores buscados no título, porém sua abordagem não atendia ao objetivo do presente estudo, ou seja, referiam-se à CIF, mas não em contexto escolar inclusivo.

\section{Resultados}

Dos 91 estudos selecionados nas três bases de dados, foram inicialmente excluídos 29 artigos por estarem repetidos, em pelo menos, duas bases de dados e/ou na mesma base de dados, utilizando-se descritores diferentes, quais sejam CIF, CIF-CJ e/ou Classificação Internacional de Funcionalidade, restando 50 artigos; destes foram excluídos 46 por não contemplarem a temática definida para o presente estudo, como por exemplo, artigos voltados à área da saúde e também repetidos, mas como já haviam sido excluídos pela temática, não foram contabilizados.

Após seguir os critérios de inclusão e exclusão, 16 artigos preencheram os critérios definidos e constituíram o corpus da presente revisão. Os artigos foram lidos na íntegra, e sua análise, dados os objetivos dos estudos, permitiu classificá-los em três categorias: artigos de revisão, artigos que abordam a utilização da CIF no contexto escolar e artigos que apresentam ferramentas e ou escopo de ferramentas e core-sets. As categorias foram definidas a posteriori em função dos objetivos dos estudos e da frequência com que apareceram.

Figura 3 - Etapa de avaliação e seleção dos trabalhos

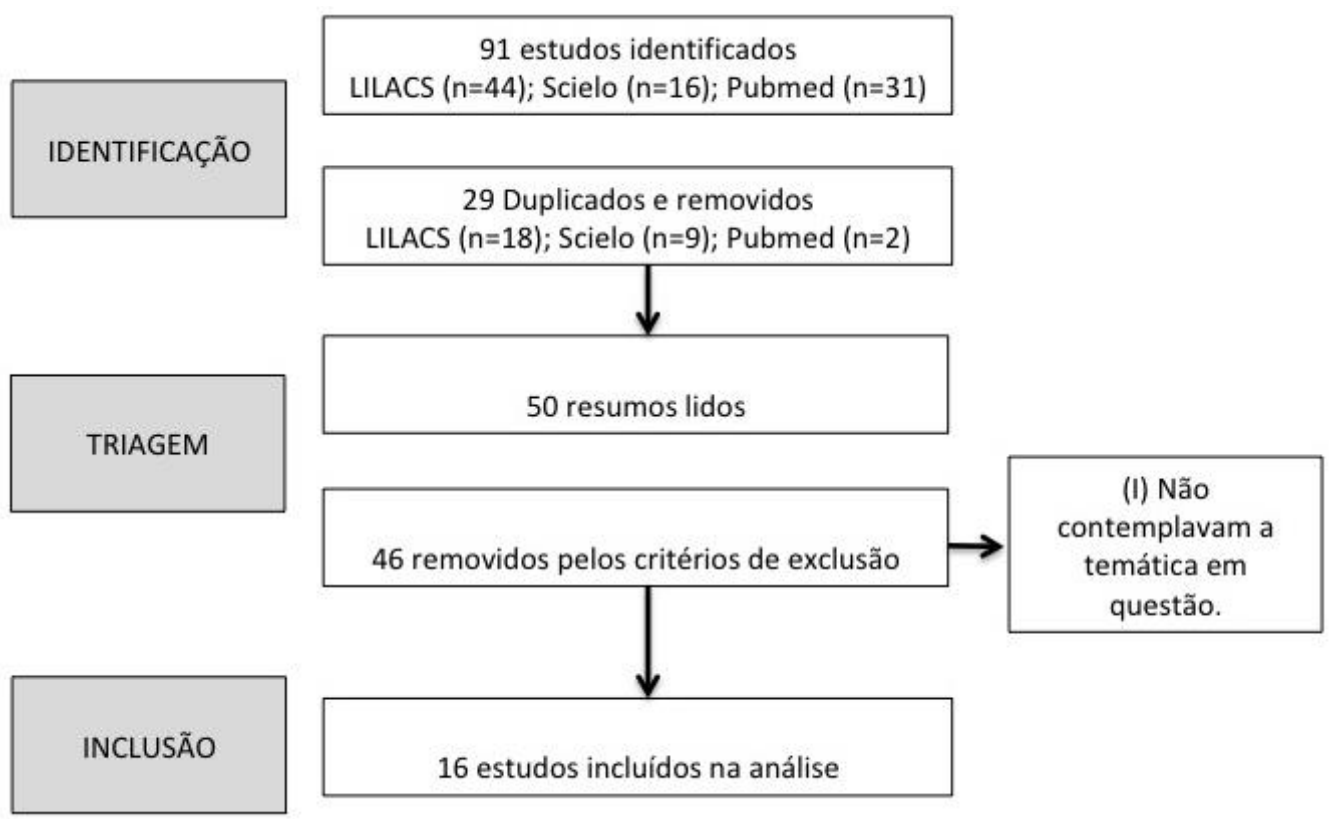

Fonte: elaborado pelas autoras (2019). 
http://dx.doi.org/10.5902/1984686X42725

A Figura 4, abaixo, descreve a frequência dos artigos no período pesquisado de 2010 a 2019. Como se pode observar, o ano 2012 foi o que apresentou a maior quantidade de artigos sobre a temática. Nos anos 2014 e 2016, não foram encontrados trabalhos relacionados ao tema.

Figura 4 - Frequência do número de publicações no período de 2010 a 2019

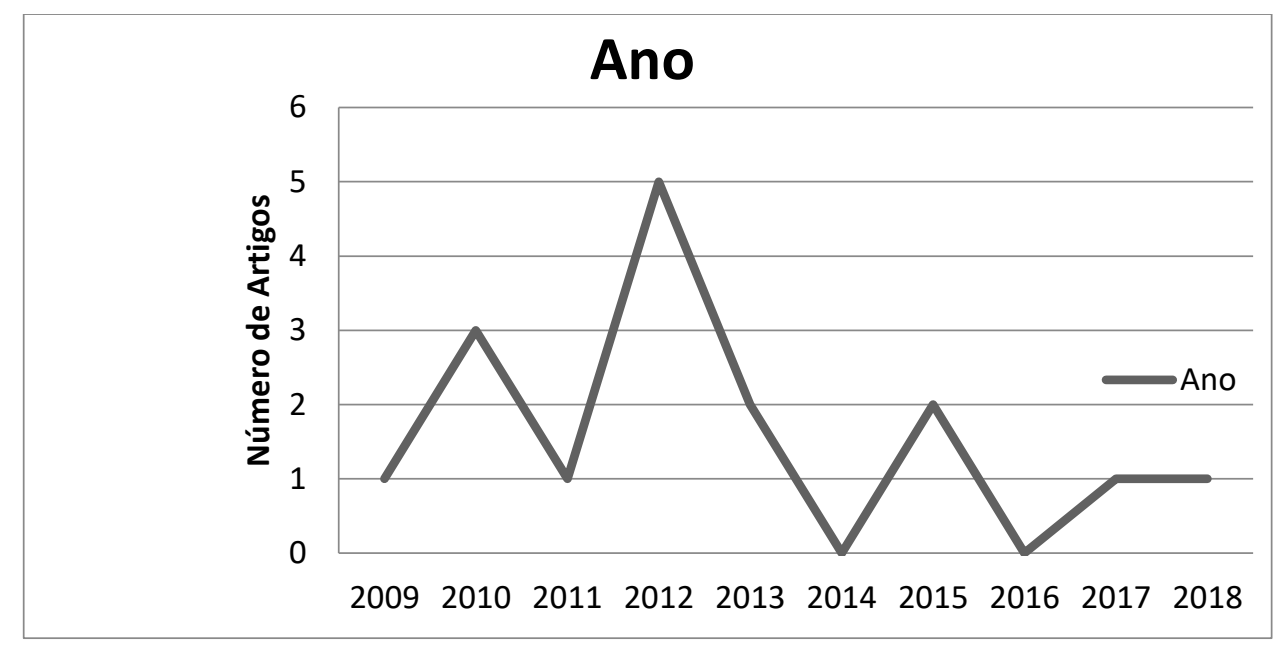

Fonte: elaborado pelas autoras (2019).

Quadro 2 - Artigos analisados

(continua)

\begin{tabular}{|c|c|c|c|c|}
\hline Ano & $\begin{array}{l}\text { Base de } \\
\text { Dados }\end{array}$ & Autores & Palavras-chave & País \\
\hline 2009 & Scielo & $\begin{array}{c}\text { Rosario, H., Leal, T., } \\
\text { Pinto, A. I., Simeonsson, } \\
\text { R. J. }\end{array}$ & $\begin{array}{c}\text { Funcionalidade, } \\
\text { classificação, intervenção } \\
\text { precoce, educação especial }\end{array}$ & Portugal \\
\hline 2010 & Pubmed & Hollenweger J. & - & Itália \\
\hline 2010 & Scielo & Rubio, M.H. & $\begin{array}{l}\text { Clasificación internacional del } \\
\text { funcionamiento de la } \\
\text { discapacidad de la salud, } \\
\text { trastornos sensoriales }\end{array}$ & Colômbia \\
\hline 2010 & Scielo & $\begin{array}{c}\text { Siqueira, I.M., Santana, } \\
\text { C.S. }\end{array}$ & $\begin{array}{c}\text { Inclusão educacional; } \\
\text { educação das pessoas com } \\
\text { deficiência; condições de } \\
\text { acesso e permanência na } \\
\text { escola }\end{array}$ & Brasil \\
\hline 2011 & Lilacs & $\begin{array}{c}\text { Miccas, C. D'Antino, } \\
\text { M.E.F. }\end{array}$ & $\begin{array}{l}\text { Pessoas com deficiência } \\
\text { mental, síndrome de Down, } \\
\text { ensino, avaliação }\end{array}$ & Brasil \\
\hline 2012 & Pubmed & $\begin{array}{l}\text { Maxwell G, Alves I, } \\
\text { Granlund M. }\end{array}$ & $\begin{array}{l}\text { Participation; environment; } \\
\text { ICF; ICF-CY; disability; } \\
\text { education. }\end{array}$ & Suécia \\
\hline
\end{tabular}


Quadro 2 - Artigos analisados

(conclusão)

\begin{tabular}{|c|c|c|c|c|}
\hline Ano & $\begin{array}{l}\text { Base de } \\
\text { Dados }\end{array}$ & Autores & Palavras-chave & País \\
\hline 2012 & Pubmed & $\begin{array}{c}\text { Sanches-Ferreira M, } \\
\text { Simeonsson RJ, } \\
\text { Silveira-Maia M, Alves } \\
\text { S, Tavares A \& Pinheiro } \\
\text { S. }\end{array}$ & $\begin{array}{l}\text { Children with disabilities; } \\
\text { functioning; ICF; legislation; } \\
\text { profile; special education. }\end{array}$ & Portugal \\
\hline 2012 & Pubmed & $\begin{array}{c}\text { Hollenweger J, Moretti } \\
\text { M. }\end{array}$ & $\begin{array}{l}\text { Eligibility; Education; } \\
\text { Disability; Diversity. }\end{array}$ & Suíça \\
\hline 2012 & Pubmed & $\begin{array}{l}\text { Moretti M, Alves I, } \\
\text { Maxwell G. }\end{array}$ & $\begin{array}{l}\text { ICF; ICF-CY; Education; } \\
\text { Special Education }\end{array}$ & Suíça \\
\hline 2012 & Pubmed & $\begin{array}{c}\text { Maxwell G, } \\
\text { Koutsogeorgou E. }\end{array}$ & $\begin{array}{c}\text { Mainstreaming (Education); } \\
\text { Social Networks; World } \\
\text { Health Organization; Public } \\
\text { Policy }\end{array}$ & Suécia \\
\hline 2013 & Pubmed & Hollenweger J. & $\begin{array}{c}\text { ICF; education; knowledge } \\
\text { management. }\end{array}$ & Suíça \\
\hline 2013 & Scielo & $\begin{array}{l}\text { Bernal-Castro, C.A., } \\
\text { Moreno-Angarita, M. }\end{array}$ & $\begin{array}{l}\text { Discapacidad intelectual, } \\
\text { clasificación, educación, } \\
\text { niños, adolescentes }\end{array}$ & Colômbia \\
\hline 2015 & Pubmed & $\begin{array}{c}\text { Adolfsson M, } \\
\text { Simmeborn Fleischer A. }\end{array}$ & $\begin{array}{l}\text { Asperger syndrome; ICF; } \\
\text { higher education; } \\
\text { requirement; student; support }\end{array}$ & Suécia \\
\hline 2015 & Scielo & $\begin{array}{l}\text { Felizola, O.L.P., } \\
\text { Esquivel, E.I.P. }\end{array}$ & $\begin{array}{c}\text { Niños con discapacidad; } \\
\text { evaluación de la } \\
\text { discapacidad; integración a la } \\
\text { comunidad; Clasificación } \\
\text { internacional del } \\
\text { funcionamiento, de la } \\
\text { discapacidad y de la salud }\end{array}$ & Colômbia \\
\hline 2017 & Scielo & Bagnato, M.J. & $\begin{array}{l}\text { Inclusión, educación terciaria, } \\
\text { discapacidad }\end{array}$ & Brasil \\
\hline 2018 & Scielo & $\begin{array}{l}\text { Andrade, M.M.A., } \\
\text { Araújo, R.C.T. }\end{array}$ & $\begin{array}{c}\text { Educação Especial, } \\
\text { deficiência, desempenho }\end{array}$ & Brasil \\
\hline
\end{tabular}

Fonte: elaborado pelas autoras (2019).

Apresentamos, a seguir, informações sobre os 16 artigos que compuseram o corpus do presente trabalho, identificando-os nominalmente e o(s) seu(s) autor(es), ano de publicação e objetivos dos estudos, conforme explicitados pelo(s) autor(es); a base de dados de localização dos artigos pode ser consultada no quadro acima. 
(continua)

\section{Título do artigo}

Avaliação de funcionalidade e participação de alunos com deficiência intelectual: estudo-piloto para elaboração de protocolo escolar (MICCAS; D'ANTINO, 2011).

Aplicación de sistemas de clasificación en contextos educativos: facilitando los procesos de inclusión de personas en situación de discapacidad intelectual (BERNAL-CASTRO;

MORENO-

ANGARITA, 2012).

Propostas de acessibilidade para a inclusão de pessoas com deficiências no ensino superior (SIQUEIRA; SANTANA, 2010).

Características de Alunos com Deficiência Física na Percepção de Seus Professores: um estudo sob os Parâmetros Conceituais da Classificação Internacional de Funcionalidade, Incapacidade e Saúde (ANDRADE; ARAÚJO, 2018).

El Desempeño Sensorial de Un Grupo de Pre-Escolares Y Escolares con Dificultades en Las Actividades Cotidianas (RUBIO, 2010).

Utilidade da Classificação Internacional da Funcionalidade, Incapacidade e Saúde: Versão para Crianças e Jovens (CIF-CJ) no Contexto da Intervenção Precoce e da Educação Especial (ROSÁRIO; PINTO; SIMEONSSON, 2009)

El niño con discapacidad: elementos orientadores para su inclusión social (ESQUIVEL; FELIZZOLA, 2015)

La inclusión educativa en la enseñanza superior: retos y demandas (BAGNATO, 2017).

Applying the ICF to identify requirements for students with Asperger syndrome in higher education (ADOLFSSON; SIMMERBORN, 2013).

Developing applications of the ICF in education systems: addressing issues of knowledge creation, management, and transfer (HOLLENWEGER, 2013).

Participation and environmental aspects in education and the ICF and the ICF-CY: Findings from a systematic literature review (MAXWELL; ALVES; GRANLUND, 2012).

\section{Objetivo do estudo}

Apresenta resultados de pesquisa que visou a elaboração inicial de um protocolo, baseado na Classificação Internacional de Funcionalidade, Incapacidade e Saúde (CIF), para avaliação de escolares com deficiência intelectual e verificar a sua aplicabilidade. Apresenta, aos professores da Colômbia, que trabalham com crianças com deficiência intelectual, ferramentas teóricas e práticas baseadas na CIF-CJ;

as autoras não apresentam o instrumento, apenas descrevem o processo de elaboração do mesmo por meio de fotos.

Artigo de revisão de projetos de inclusão que utilizou a CIF para organizar e classificar os dados obtidos na leitura dos projetos.

Objetivou identificar como os professores caracterizam a deficiência física de seus alunos e discutir sobre possíveis implicações das tendências perceptivas envolvidas nessa caracterização; o roteiro da entrevista desta pesquisa foi baseada em três categorias da CIF, sendo elas: funções e estruturas corporais, atividade e participação e fatores ambientais

Estudo da Colômbia em que são avaliados os processamentos sensoriais através da escolha de itens da CIF para avaliar diversos aspectos sensoriais, sem, no entanto, apresentar o Core Set utilizado

Artigo português de revisão que apresenta estudo da implementação da CIF-CJ nos processos de intervenção precoce, para além do diagnóstico, apresentando instrumentos que falam a mesma língua da CIF.

Discute as formas como os professores caracterizam os alunos com deficiência física, comparando-se com os conceitos apresentados pela CIF.

Discute conceitos teóricos a respeito da inclusão de alunos com deficiência no ensino superior.

Visou identificar as categorias da CIF que refletem as necessidades diárias da vida do estudante com Asperger para que se desenvolva uma ferramenta estruturada para guiar diálogos entre coordenadores e estudantes com Asperger nas Universidades da Suécia

Apresenta como a CIF pode ser utilizada no sistema educacional para auxiliar equipes multidisciplinares em aquisição do conhecimento e mapeamento do que já existe, bem como criar conhecimentos e novas aplicações em propostas específicas

Objetivo foi analisar e investigar a aplicação do domínio participação na pesquisa sobre educação através de uma revisão sistemática. Especificamente, como a participação está relacionada às dimensões ambientais: disponibilidade, acessibilidade, acomodação e aceitabilidade 
http://dx.doi.org/10.5902/1984686X42725

Quadro 3 - Informativo sobre os estudos

(conclusão)

\begin{tabular}{|c|c|}
\hline Título do artigo & Objetivo do estudo \\
\hline $\begin{array}{l}\text { Development of an ICF-based eligibility } \\
\text { procedure for education in Switzerland } \\
\text { (HOLLENWEGER, 2010). }\end{array}$ & $\begin{array}{l}\text { Fornece uma visão geral das diferentes práticas } \\
\text { relacionadas à elegibilidade, com foco particular em } \\
\text { crianças com deficiência, mostrando que em } 2011 \text { a } \\
\text { Suíça implementaria um procedimento multidimensional } \\
\text { para estabelecer a elegibilidade nos sistemas } \\
\text { educacionais. Descreve a estrutura filosófica e conceitual } \\
\text { do procedimento baseado na CIF e na Convenção das } \\
\text { Nações Unidas sobre os Direitos das Pessoas com } \\
\text { Deficiência. }\end{array}$ \\
\hline $\begin{array}{l}\text { Portugal's special education law: } \\
\text { implementing the International Classification } \\
\text { of Functioning, Disability and Health in policy } \\
\text { and practice (SANCHEZ-FERREIRA et al., } \\
\text { 2012). }\end{array}$ & $\begin{array}{l}\text { A CIF foi introduzida na lei da educação em Portugal } \\
\text { como o sistema obrigatório para orientar a política e a } \\
\text { prática de elegibilidade na Educação Especial, e o artigo } \\
\text { descreve sua implementação. }\end{array}$ \\
\hline $\begin{array}{l}\text { Using the International Classification of } \\
\text { Functioning, Disability and Health } \\
\text { Childrenand Youth Version in Education } \\
\text { Systems (HOLLENWEGER; MORETTI, } \\
\text { 2012). }\end{array}$ & $\begin{array}{l}\text { Apresenta a CIF como modelo de classificação, os } \\
\text { diferentes fatores que influenciam as decisões } \\
\text { relacionadas à elegibilidade (deficiências, } \\
\text { atividade/participação, ambiente, fatores pessoais) que } \\
\text { pode se tornar transparente para fornecer base para um } \\
\text { processo de tomada de decisão. }\end{array}$ \\
\hline $\begin{array}{l}\text { A systematic literature review of the situation } \\
\text { of the International Classification of } \\
\text { Functioning, Disability, and Health and the } \\
\text { International Classification of Functioning, } \\
\text { Disability, and Health-Children and Youth } \\
\text { version in education: a useful tool or a flight } \\
\text { of fancy? (MORETTI; ALVES; MAXWELL, } \\
\text { 2012). }\end{array}$ & $\begin{array}{l}\text { Apresenta o resultado de uma revisão sistemática que } \\
\text { explora a aplicabilidade da CIF e da CIF-CJ em diversos } \\
\text { níveis e em processos dos sistemas educacionais em } \\
\text { diversos países. }\end{array}$ \\
\hline $\begin{array}{l}\text { Using social capital to construct a } \\
\text { conceptual International Classification of } \\
\text { Functioning, Disability and Health Children } \\
\text { and Youth version-based framework for } \\
\text { stronger inclusive education policies in } \\
\text { Europe (MAXWELL; KOUTSOGEORGOU, } \\
\text { 2012). }\end{array}$ & $\begin{array}{l}\text { Analisa o funcionamento de políticas e sistemas de } \\
\text { educação inclusiva e propor políticas educacionais } \\
\text { inclusivas mais fortes por meio da combinação de capital } \\
\text { social e políticas de educação inclusivautilizando a CIF. }\end{array}$ \\
\hline
\end{tabular}

Fonte: elaborado pelas autoras (2021).

\section{Discussão e considerações finais}

Com o presente estudo objetivou-se realizar um mapeamento bibliográfico da literatura acerca da operacionalização da CIF no contexto escolar inclusivo, e compreender como a mesma está sendo utilizada no Brasil e em outros países.

A CIF possibilita avaliar diferentes atributos do indivíduo nas dimensões de funções e estruturas do corpo, atividade e participação, levando-se em consideração a ação de fatores contextuais e ambientais; permite coletar dados vitais (DI NUBILA, 2010) sobre tipos e níveis de funcionalidade e incapacidade de modo consistente e comparável 
http://dx.doi.org/10.5902/1984686X42725

internacionalmente, fornecendo a base para compor dados em nível nacional, ajudando a guiar o desenvolvimento de políticas nessa área.

É uma ferramenta útil para conhecer as condições da funcionalidade das pessoas, associadas ou não à qualquer doença, bem como identificar os fatores ambientais e pessoais que favoreçam suas atividades e, consequentemente, sua qualidade de vida (ARAÚJO, 2015).

Conforme Ruaro et al. (2012), a CIF tem sido apontada como uma ferramenta multidimensional que visualiza a saúde humana sob diversas vertentes e abordagens, podendo ser usada nos mais variados setores, incluindo a educação. Sua utilização deve ser implementada em práticas clínicas, no ensino e em pesquisas.

Rosário et al. (2009) relatam que a CIF ultrapassa as limitações da abordagem diagnóstica do desenvolvimento, descrevendo características da funcionalidade individual e considerando a participação da pessoa em processos interativos com o ambiente. A avaliação abrangente da funcionalidade é essencial para a elaboração de intervenções que sirvam de base para a redução de limitações e o aumento do bem-estar das crianças.

É quase inexistente as investigações que tratam da importância, da CIF na Educação inclusiva, especialmente no que se refere à aproximação das possibilidades e dificuldades apresentadas pelos alunos com deficiência inseridos no contexto escolar. Sabe-se que o diagnóstico por si não prevê a descrição do sujeito em seu estado funcional, tampouco o tipo e nível de apoios e cuidados que cada sujeito requer para desenvolver seu potencial de desenvolvimento e aprendizagem.

A escolha pela CIF dá-se porque esta é uma classificação capaz de avaliar o aluno de modo não reducionista, sendo capaz de responder às necessidades de avaliação com vistas às intervenções. Outro ponto importante é que a CIF pode oferecer subsídios para a previsão e provisão de requisitos específicos de cada criança com deficiência, em um determinado contexto educacional, em que muitas variáveis relevantes contextuais podem ser consideradas, bem como diferentes fontes de informação podem ser consideradas, como os pontos de vista de professores e pais (HOLLENWEGER, 2013).

O modelo teórico, ou ainda, mesmo o ideológico da educação especial com perspectiva na educação inclusiva está presente em todas as esferas legais, contudo, esses indivíduos precisam ser respeitados em suas individualidades para que assim se desenvolvam em um ambiente saudável e acolhedor, capaz de auxiliar em sua autonomia. Sendo assim, acreditamos que é de suma importância a implementação de políticas 
públicas baseadas no constructo da CIF, com uma visão integradora do sujeito, no âmbito educacional brasileiro. Os alunos com deficiência, matriculados no sistema regular de ensino, tendo como perspectiva a educação inclusiva, poderão ser beneficiados com um sistema de avaliação que tem por foco a funcionalidade, pautado nos preceitos da CIF, uma vez que o conhecimento sobre suas demandas educacionais específicas poderá servir de base segura para a elaboração e implantação de programas de ensino individualizado.

O estudo de Maia e Lopes-dos-Santos (2010), embora não identificado nas bases de dados deste estudo, indicou que as documentações de alunos com deficiência, elaborados em referência à CIF-CJ, apresentam mais indicadores de funcionalidade. Este estudo mostrou ainda que o uso da CIF-CJ indicou uma promoção dos aspectos ambientais nos relatórios dos professores, identificando aquilo que pode ser melhorado ou criado no ambiente para promover a participação dos alunos com deficiência de forma mais eficaz. Outro estudo, também não localizado nas bases de dados, de autoria de Castro e Grande (2018), mapeou o Early Development Instument (EDI) com a CIF-CJ, e identificou as dimensões de funcionamento consideradas por especialistas como essenciais para a idade de 3 a 5 anos, tendo concluído que o EDI deve complementar outras medidas com enfoque nas Funções Corporais e Fatores Ambientais, a fim de facilitar uma descrição holística da criança. São estudos, assim como possivelmente outros, que denotam que a CIF tem sido estudada e aplicada em contexto educativo regular e na educação especial em outros países. Cabe aqui considerar que dos 16 artigos que compuseram o corpus deste estudo, apenas 04 são brasileiros, o que vale dizer que estamos, ainda, engatinhando se observarmos que destes estudos, 02 foram publicados, respectivamente, em 2010 e 2011, e 02, em 2017 e 2018.

O hiato observado em produção brasileira, ligada à temática em questão, aponta a escassez de estudos que visem compreender o alunado público-alvo da educação especial inclusiva, numa perspectiva biopsicossocial. Assim, pode-se pensar que tal alunado pode estar sendo concebido, no âmbito escolar, a partir de sua deficiência e não em função de sua real funcionalidade. Avaliar alunos com deficiência "incluídos" na escola regular deveria pressupor uma mudança paradigmática no conceito de deficiência, justamente aquela proposta pela CIF. 
A maior contribuição do constructo teórico da CIF nos parece recair justamente no conceito de deficiência que apresenta, ou seja, coloca a funcionalidade do sujeito à frente de sua condição de deficiência.

A CIF, assim, ultrapassa a abordagem diagnóstica da CID-10, descrevendo características da funcionalidade do indivíduo, considerando a atividade e participação deste em processos interativos com o ambiente. Miccas, D’Antino \& Vital (2014, n.p.) acreditam que, a despeito da importância de um diagnóstico clínico, o conhecimento da funcionalidade de cada indivíduo seja fundamental para que se possam desenhar sistemas de apoio educacional adequados a cada caso. Tem-se, assim, o enfoque na funcionalidade humana e não apenas nas incapacidades da pessoa, levando em conta todo um contexto no qual o sujeito está inserido, considerando os fatores contextuais que influenciam o seu desempenho e sua qualidade de vida.

Assim, no contexto da educação inclusiva brasileira, a compreensão, pelos professores e demais atores educacionais, do conceito de pessoa com deficiência na perspectiva da funcionalidade humana, derivada de fatores ambientais, dentre os quais a escola se insere, poderá mudar substancialmente propostas pedagógicas que visem contribuir de forma efetiva com a dimensão funcional de cada educando, atendendo suas demandas específicas.

\section{Referências}

ADOLFSSON, Margareta; SIMMEBORN, Ann Fleischer. Applying the ICF to identify requirements for students with Asperger syndrome in higher education. Developmental Neurorehabilitation, v. 18, p. 190-202, Agosto 2013.

ANDRADE, Mirela Moreno de Almeida; ARAÚJO, Rita de Cássia Tibério. Características de Alunos com Deficiência Física na Percepção de Seus Professores: um estudo sob os Parâmetros Conceituais da Classificação Internacional de Funcionalidade, Incapacidade e Saúde. Revista Brasileira de Educação Especial, v. 24, n. 1, p. 3-16, 2018.

ARAÚJO, Eduardo Santana; BUCHALLA, Cassia Maria. The use of the International Classification of Functioning, Disability and Health in health surveys: a reflexion on its limits and possibilities. Rev Bras Epidemiol, p. 720-724, JUL-SEP, 2015.

BAGNATO, Maria José. La inclusión educativa en la enseñanza superior: retos y demandas. Educar em Revista, n. especial 3, p. 15-26, 2017.

BERNAL-CASTRO, Carol Andrea; MORENO-ANGARITA, Marisol. Aplicación de sistemas de clasificación en contextos educativos: facilitando los procesos de inclusión de personas en situación de discapacidad intelectual. Revista De La Universidad De Medicina, v. 61, n.2, p. 123-135, 2012. 
http://dx.doi.org/10.5902/1984686X42725

BRASIL. Ministério da Educação. Nota Técnica № 24/ 2013/ MEC/ SECADI/

DPEE. Orientação aos Sistemas de Ensino para a implementação da Lei nํ⒓764/2012.

Disponível em:

http://portal.mec.gov.br/index.php?option=com_docman\&view=download\&alias=13287-

nt24-sistem-lei12764-2012\&category_slug=junho-2013-pdf\&Itemid=30192. Acesso em: 20 de junho de 2018.

BRASILEIRO, Ismênia de Carvalho; MOREIRA, Thereza Maria Magalhães; JORGE, Maria Salete Bessa. Interveniência dos fatores ambientais na vida de crianças com paralisia cerebral. Acta Fisiátrica, v. 16, n. 3, p. 132-137, 2009.

CASTRO, Susana; PINTO, Ana Isabel. Identification of core functioning features for assessment and intervention in Autism Spectrum Disorders. Disability \& Rehabilitation, v. 2 , n. 35 , p. 2012.

CASTRO, Suzana; GRANDE, Catarina. Linking the early development instrument with the ICF-CY. International Journal of Developmental Desabilites, v. 64, n. 1. p. 3-15, 2018.

DI NUBILA, Heloisa Brunow Ventura. Uma introdução à CIF - Classificação Internacional de Funcionalidade, Incapacidade e Saúde. Revista Brasileira de Saúde Ocupacional, v. 35, n. 121, p. 122-123, junho. 2010.

DI NUBILA, Heloisa Brunow Ventura; BUCHALLA, Cássia Maria. O papel das Classificações da OMS - CID e CIF nas definições de deficiência e incapacidade. Revista Brasileira de Epidemiologia, v. 11, n.2, p. 324-35. 2008.

ESQUIVEL, Eliana Isabel Parra; FELIZZOLA, Olga Luz Peñas. El niño con discapacidad: elementos orientadores para su inclusión social. Revista Científica Salud Uninorte, v. 31, n. 2, p. 329-346, 2015.

FARIAS, Norma; BUCHALLA, Cassia Maria. A Classificação Internacional de Funcionalidade, Incapacidade e Saúde - Rev Bras Epidemiol 2005; 8(2): 187-93.

HOLLENWEGER, Judith. Development of an ICF-based eligibility procedure for education in Switzerland. BMC Public Health 11, S7 (2011) doi: 10.1186/1471-2458-11-S4-S7.

HOLLENWEGER, Judith. Developing applications of the ICF in education systems: addressing issues of knowledge creation, management and transfer. Journal Disability and Rehabilitation, v.35, n. 13, p. 1.087-91, 2013.

HOLLENWEGER, Judith; MORETTI, Marta. Using the International Classification of Functioning, Disability and Health Children and Youth version in education systems: a new approach to eligibility. American journal of physical medicine \& rehabilitation/ Association of Academic Physiatrists, v. 13, n1, p.97-102, 2012.

MAIA, Mónica Silveira; LOPES-DOS-SANTOS, Pedro. Práticas em Educação Especial à Luz do Modelo Biopsicossocial: O Uso da CIF-CJ como Referencial na Elaboração dos Programas Educativos Individuais. Actas do VII Simpósio Nacional de Investigação em Psicologia Universidade do Minho, Portugal, 2010. 
MAXWELL, Gregor; ALVES Ines; GRANLUND, Mats. Participation and environmental aspects in education and the ICF and the ICF-CY: findings from a systematic literature review. Journal Developmental Neurorehabilitation, v. 15, n. 1, p. 63-78, 2012.

MAXWELL, Gregor; KOUTSOGEORGOU, Eleni. Using social capital to construct a conceptual International Classification of Functioning, Disability, and Health Children and Youth version-based framework for stronger inclusive education policies in Europe. American journal of physical medicine \& rehabilitation/ Association of Academic Physiatrists, v. 91, n.13, p. 118-123, 2012.

MICCAS, Camila; D’ANTINO, Maria Eloisa Famá. Avaliação de funcionalidade em atividades e participação de alunos com deficiência mental: estudo piloto para elaboração de protocolo escolar. Temas sobre Desenvolvimento, v. 18, n. 102, p. 82-95, 2011.

MORETTI, Marta; ALVES, Ines; MAXWELL, Gregor. A systematic literature review of the situation of the International Classification of Functioning, Disability and Health and the International Classification of Functioning, Disability, and Health-Children and Youth version in education: a useful tool or a flight of fancy? American journal of physical medicine \& rehabilitation/Association of Academic Physiatrists, v. 91, n.13, p. 113117, 2012.

ORGANIZAÇÃO MUNDIAL DA SAÚDE (OMS). Classificação Internacional de Funcionalidade, Incapacidade e Saúde. São Paulo, Edusp, 2008.

ORGANIZAÇÃO MUNDIAL DA SAÚDE (OMS). Classificação Internacional de Funcionalidade, Incapacidade e Saúde: versão para Crianças e Jovens. São Paulo: Edusp, 2011.

PORTUGAL. DL nº 3/2008, 7 de janeiro de 2008. Direção geral da Educação de Portugal.

RIBERTO, Marcelo. Core sets da Classificação Internacional de Funcionalidade, Incapacidade e Saúde. Revista Brasileira de Enfermagem, v. 64, n. 5, p. 938-946, 2011.

ROSÁRIO, Helena; LEAL, Isabel. PINTO, Ana Isabel. SIMEONSSON, Rune. Utilidade da Classificação Internacional da Funcionalidade, Incapacidade e Saúde: versão para crianças e jovens (CIF-CJ) no contexto da intervenção precoce e da educação especial. Psicologia, v. 23, n. 2, p. 129-139, 2009.

RUARO, João Afonso; RUARO, Marinêz; SOUZA, Damião; FRÉZ, Andersom; GUERRA, Ricardo. Panorama e perfil da utilização da CIF no Brasil - uma década de história. Revista Brasileira de Fisioterapia, v. 16, n.6, p. 454-462, 2012.

RUBIO, Maria Helena. El Desempeño Sensorial de Un Grupo de Pre-Escolares y Escolares Con Dificultades En Las Actividades Cotidianas. Revista De La Universidad De Medicina, v. 58, n. 4, p. 283-292, 2010.

SANCHES-FERREIRA, Manuela; SIMEONSSON, Rune; SILVEIRA-MAIA, Mónica; ALVES, Sílvia; TAVARES, Ana; PINHEIRO, Sara. Portugal's special education law: implementing the International Classification of Functioning, disability and Health in policy and practice. Journal Disability and Rehabilitation, v. 35, n. 10, p. 868-873, 2012. 
http://dx.doi.org/10.5902/1984686X42725

SEKKEL, Marie Claire. A construção de um ambiente inclusivo na educação infantil: relato e reflexão sobre uma experiência. Tese de Doutorado em Psicologia, Instituto de Psicologia, Universidade de São Paulo, São Paulo - 2003.

SIQUEIRA, Inajara Mills; SANTANA, Carla da Silva. Propostas de Acessibilidade para a Inclusão de Pessoas com Deficiências no Ensino Superior. Revista Brasileira de Educação Especial, v.16, n.1, p.127-136, 2010.

STUCKI, Gerold, et al. O desenvolvimento da "Pesquisa em Funcionalidade Humana e Reabilitação" a partir de uma perspectiva abrangente - ACTA FISIATR 2008; 15(1): 63 69.

VELTRONE, Aline Aparecida; MENDES, Enicéia. Gonçalves. Descrição das propostas do Ministério da Educação na avaliação da deficiência intelectual. Paidéia, v. 21, n. 50, p. 413-421, 2011.

\section{Notas}

1 Em 2015 acontece uma reedição da CIF, onde passa a ser integrada à versão anterior da CIF-CJ e esta por sua vez é extinta, porém como o estudo era um mapeamento bibliográfico dos últimos anos, as autoras não poderiam deixar de considerar a CIF-CJ nos estudos.

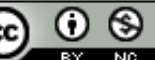

This work is licensed under a Creative Commons Attribution-NonCommercial 4.0 International (CC BY-NC 4.0) 